\title{
Définitions des règles de modélisation thermique des machines électriques tournantes
}

\author{
D. Roye et R. Perret \\ Laboratoire d'Electrotechnique de Grenoble (*), ENSIEG, Institut National Polytechnique de Grenoble, \\ BP 46, 38402 Saint Martin d'Hères, France
}

(Reçu le 17 mai 1984, révisé le 26 novembre, accepté le 29 novembre 1984)

\begin{abstract}
Résumé. - Dans cet article, les auteurs définissent les règles d'une modélisation thermique des machines électriques tournantes permettant la détermination des échauffements lorsque les pertes sont connues. Ils présentent une adaptation de la méthode nodale. Ils décrivent les principes de choix du découpage en blocs élémentaires et de la méthode de modélisation. Enfin ils donnent les formules permettant le calcul des coefficients d'échanges dans les conditions de fonctionnement des machines classiques et un exemple de simulation.
\end{abstract}

\begin{abstract}
In this paper the authors define the rules of a thermal modelling of rotating electrical machines which permits the determination of machine heating when losses are known. An adaptation of the nodal method is presented. The principles of partition into elementary blocks and the modelling method are described. Finally, formulae permitting the calculation of coefficients of exchange under operating conditions of classical machines are given and an example of simulation is presented.
\end{abstract}

L'évolution dans la construction des machines a entraîné une augmentation appréciable des puissances massiques avec comme conséquence un affaiblissement des constantes de temps thermiques et donc une plus grande sensibilité potentielle aux surcharges calorifiques [1 à 13]. L'utilisation avec des alimentations non traditionnelles qui entraînent des modifications des répartitions et des valeurs des pertes nécessite trop souvent alors un déclassement, le dimensionnement et les indications nominales correspondant toujours aux types d'alimentations classiques, sinusoïdales ou parfaitement continues [16-18].

La démarche expérimentale seule, même si elle paraît souvent la plus précise, ne peut suffire. La diversité des fonctionnements, la dispersion des caractéristiques, l'inaccessibilité de certaines informations, conduiraient alors à une procédure sans doute longue, onéreuse et incomplète. La simulation des comportements thermiques associée à des vérifications expérimentales présente une souplesse d'utilisation qui semble mieux adaptée à de telles investigations [19].
Dans cet article, nous proposons une modélisation des transferts thermiques entre les différents éléments d'une machine afin de déduire l'échauffement correspondant à une répartition de pertes connues.

Une machine électrique tournante présente une très forte hétérogénéité thermique, conséquence des propriétés des différents matériaux qui la constituent, mais sa géométrie est régulière, montrant de nombreuses symétries et pouvant se ramener souvent à des formes simples, cylindriques, planes ou hémisphériques. Une discrétisation relativement grossière qui réduit le découpage à un nombre limité d'éléments de formes géométriques simples, pour tout le volume de la machine est alors possible.

Le nombre des formes prévisibles étant limité, le nombre de modèles élémentaires l'est aussi et la modélisation générale ne s'en trouve pas compliquée.

Cette démarche offre l'avantage de permettre de bénéficier en partie des résultats d'études, parfois anciennes, fournissant des exemples de modèles thermiques simplifiés, résolus souvent analytiquement, valables pour des formes géométriques précises.

Les règles essentielles de la modélisation retenue sont exposées dans les paragraphes suivants, à travers

(*) CNRS LA 355. 
la description des grandes étapes de la réalisation de cette modélisation :

- définition d'une géométrie simplifiée et découpage de la machine en blocs élémentaires;

- établissement pour chaque bloc des équations de bilan des flux de chaleur et des conditions aux limites : définition des modèles élémentaires;

- détermination des coefficients intervenant dans ces différentes relations.

\section{Définition d'une géométrie simplifiée et découpage de la machine.}

1.1 PRINCIPE DE LA DÉCOMPOSITION EN BLOCS. Toute la valeur du modèle repose sur la quálité du découpage en blocs. Ces blocs doivent tous présenter une unité suffisante, tant du point de vue géométrique que du point de vue physique et thermique :

- l'unité géométrique se traduit par une forme découpée se ramenant à un volume simple : par exemple secteur de cylindre ou de sphère (plein ou creux), parallélépipède, ....,

- l'unité physique se traduit par l'homogénéité du corps remplissant le volume ou la régularité et la finesse de sa structure en cas de corps composé,

- l'unité thermique se traduit par l'unicité du mode de transfert prépondérant et l'uniformité de la production de chaleur, quand elle existe.

Il y a, bien sûr, une très forte corrélation entre découpage et structure, les directions de celui-ci devant correspondre aux éventuelles directions d'anisotropie de celle-là.

A cette unité formelle et structurelle, que nous venons de définir, s'ajoute également, comme guide pour le découpage, afin de garantir tout intérêt pour l'utilisation du modèle, une unité fonctionnelle se traduisant par l'importance de la partie délimitée par le bloc dans le fonctionnement de la machine.

La machine simplifiée est alors constituée de la juxtaposition de blocs de formes différentes dans lesquels la chaleur est produite et transférée par conduction. Ces blocs délimitent des cavités dans lesquelles la chaleur est transmise d'une surface à une autre par conduction à travers un fluide, ici l'air, par convection et par rayonnement [20]. L'ensemble est entouré d'une enceinte, vers laquelle les surfaces extérieures des blocs périphériques rayonnent, et baigne dans l'air ambiant auquel de la chaleur est cédée par convection (Fig. 1). Généralement l'enceinte et l'air ambiant sont considérés à une température identique, constante, prise comme référence pour les échauffements.

\subsection{Définition et Classification Des ÉlÉments} REPRÉSENTANT LES BLOCS. - A partir des blocs définis par le découpage et des cavités délimitées par les blocs, différents types d'éléments vont pouvoir être définis. Un élément du modèle thermique se caractérise essentiellement par le mode de transfert de chaleur qui s'y produit (Fig. 2).

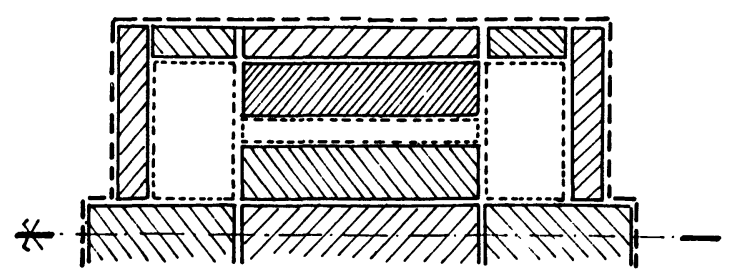

Fig. 1. - Représentation schématique et symbolique du découpage et de la définition des éléments. $\square$ Blocs conductifs. [־ Cavités. ᄃコ Milieu extérieur.

[Schematic and symbolic representation of the partition and definition of the elements. $\square$ Conditions blocks. $[:-$ ב Cavities. [コExternal medium.]

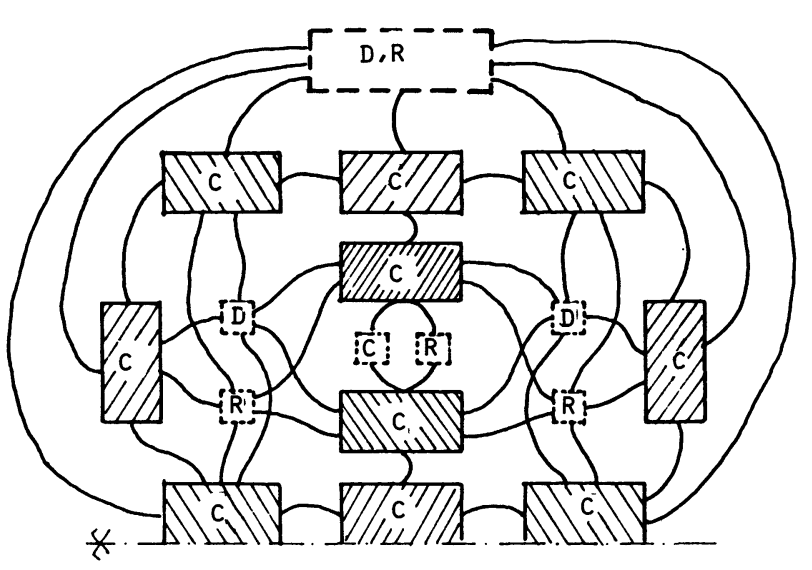

Fig. 2. - Définition des éléments du modèle et de leurs interconnexions. C : Transfert par conduction. D : Convection. $\mathbf{R}$ : Rayonnement.

[Definition of model elements and their interconnection. C : Conduction transfer. D : Convection. R : Radiation.]

Cette classification se justifie par les remarques suivantes valables pour une machine :

- le mode de transfert est généralement unique;

- lorsque plusieurs modes coexistent dans un milieu, soit l'un est très largement prépondérant, soit ils n'interfèrent pas. Ainsi tous les solides sont opaques et très absorbants, l'air est transparent, le confinement extrême du fluide dans l'entrefer favorise la conduction au détriment de la convection.

Décrire le système thermique revient alors à préciser:

- les types des éléments,

- les liaisons entre eux.

Compte tenu des critères de classification, les différents types d'éléments peuvent être, par ailleurs, regroupés en deux familles :

- les éléments pour lesquels le transfert est défini dans tout le volume, suivant différentes directions 
éventuellement. Leur définition géométrique leur est donc propre. Ce sont les éléments pour lesquels le mode de transfert est la conduction et ceux pour lesquels le mode de transfert est la convection forcée (déplacement de matière imposé dans une direction). Pour les distinguer on leur attribue, par la suite, le qualificatif de volumiques,

- les éléments pour lesquels le transfert est défini aux surfaces qui les délimitent. Leur définition géométrique dépend donc, d'un point de vue "thermique», essentiellement des éléments volumiques auxquels ils sont reliés, c'est-à-dire des surfaces d'échanges par lesquelles ils sont en contact.

Ce sont les éléments définis à partir des cavités entièrement closes dans lesquelles les transferts sont effectués entre les surfaces frontières par rayonnement et par l'intermédiaire du fluide enfermé, l'échange se faisant alors par convection libre et convection forcée, certaines surfaces pouvant être en mouvement. Pour les distinguer on leur attribue, par la suite, le qualificatif de surfaciques.

Les liaisons entre éléments sont donc fondamentales pour la définition de ceux appartenant à cette deuxième famille.

La classification distingue donc finalement cinq types d'éléments :

- Les éléments volumiques conductifs qui peuvent être uni-, bi-, ou tri-directionnels selon l'anisotropie et la configuration des éléments adjacents qui guident les transferts. Ils auront donc, suivant les cas, en général, au maximum six liaisons. Ces éléments sont comme on l'a vu, définis par leur volume dans lequel se produit le dégagement de chaleur et à travers lequel s'effectue le transfert, souvent dans des directions privilégiées.

- Les éléments volumiques convectifs dans lesquels la convection est forcée et guidée par la géométrie propre de ces éléments : tuyaux ou canaux de sections quelconques. Ils auront deux liaisons, avec des éléments du même type généralement, suivant la direction de l'écoulement auxquelles s'ajoute un nombre de liaisons égal au nombre de surfaces d'échanges définissant la géométrie de l'élément.

- Les éléments surfaciques convectifs clos dans lesquels le fluide ne sert que d'intermédiaire entre les surfaces et qui peuvent donc être très largement multidirectionnels. Rappelons que le fluide est considéré comme non absorbant.

- Les éléments surfaciques rayonnants pour lesquels les échanges s'effectuent entre surfaces et ne sont définis que par les caractéristiques de ces surfaces et leurs orientations les unes par rapport aux autres. Ils peuvent être très largement multidirectionnels.

- Le milieu ambiant pris comme élément surfacique de référence constitué en fait par l'enceinte globale et par l'air ambiant. Il est donc à la fois rayonnant et convectif. Cet élément est lui aussi largement multidirectionnel.

\section{Définition des modèles élémentaires.}

Le découpage en blocs des milieux solides et fluides de la machine reste identique, dans son principe, à celui de la méthode des différences finies.

A ce découpage et à la définition des éléments sont associées des hypothèses d'uniformité :

- uniformité des températures, à l'intérieur de l'élément (bloc ou cavité) et sur chacune des faces,

- uniformité de la variation d'énergie interne pour les éléments solides,

- uniformité de la production éventuelle de chaleur pour les éléments solides,

- uniformité de la vitesse d'écoulement pour les éléments fluides,

- uniformité des propriétés physiques pour tout élément : masse volumique, chaleur massique, conductivité thermique $(k)$ suivant chaque direction d'anisotropie et, pour le rayonnement thermique, émissivité $\left(\varepsilon_{\lambda}\right)$ sur chacune des faces,

- uniformité des conditions d'échanges par convection pour chacune des faces (coefficient d'échanges $h$ ).

Ces hypothèses amènent à considérer donc uniquement des valeurs moyennes des différentes grandeurs pour la définition des modèles élémentaires. Parmi ces hypothèses, celles concernant l'uniformité des températures et la variation d'énergie interne peuvent être les plus restrictives selon les propriétés conductrices et les formes allongées ou massives de l'élément.

A chaque élément représentant un bloc du découpage correspond, comme dans la discrétisation pour la méthode des différences finies, un nœud relié aux nœuds des éléments adjacents par un nombre fini de connexions. Dans ce cas, cependant, la température au noud de l'élément est exprimée en fonction de celles des diverses faces de l'élément. Il est donc intéressant de définir, associés à chacune de ces faces, et donc des possibilités de contact avec d'autres éléments, des noeuds, appelés en conséquence noeuds de liaisons, dont la température est la température moyenne de la face.

Par commodité, pour tous les calculs et les raisonnements, une représentation graphique, par des schémas électriques équivalents des modèles élémentaires, peut être utilisée systématiquement.

2.1 ModÉlISATION DES ÉLÉMENTS CONDUCTIFS. - La simplicité de la géométrie des éléments et la simplification des conditions aux limites (qui se ramènent toutes à des conditions de Dirichlet avec les hypothèses d'uniformité des températures sur les faces) rend possible l'établissement d'une expression analytique de la répartition de température à l'intérieur des éléments.

Compte tenu des symétries de révolution et de l'existence de directions privilégiées dans une machine pour les transferts de chaleur, une expression de la répartition unidirectionnelle ou bidirectionnelle suffit parfois et très souvent, dans le cas où une répartition multi-directionnelle s'impose, l'hypothèse d'indépen- 
dance complète des transferts suivant chacune des directions retenues s'avère suffisante. On est ainsi amené à définir un modèle thermique élémentaire unidirectionnel à partir duquel on peut extrapoler les modèles directionnels multiples et multidirectionnels.

2.1.1 Principe de la représentation unidirectionnelle. - En appelant $p$ la densité de puissance produite dans le corps, $T$ la température, $C_{\mathrm{v}}$ la chaleur massique, $\rho$ la masse volumique, nous pouvons écrire l'équation décrivant le transfert thermique dans la direction unique $x$ (Fig. 3a) (conductivité thermique $k$ dans cette direction) sous la forme :

$$
k \frac{\partial^{2} T}{\partial x^{2}}=-p+\rho C_{\mathrm{v}} \frac{\partial T}{\partial t}
$$

où $k, p, \rho$ et $C_{\mathrm{v}}$ sont des valeurs moyennes, donc indépendantes de $x$, et $\partial T / \partial t$ est considérée indépendante de $x$. L'intégration est alors aisée. On obtient ainsi une expression analytique de la répartition des températures suivant la direction $x$ qui peut se décomposer en deux termes (Fig. 3b) :

$$
T(x)=T_{\mathrm{DT}}\left(T_{1}, T_{2}, x\right)+T_{\mathrm{CP}}\left(p, \rho, C_{\mathrm{v}}, \partial T / \partial t, x\right),
$$

- le premier, $T_{\mathrm{DT}}$, traduit le transfert de chaleur à travers l'élément provoqué, par l'écart éventuel entre les températures $T_{1}$ et $T_{2}$ des deux faces de l'élément suivant la direction considérée, isothermes par hypothèse;

- le second, $T_{\mathrm{CP}}$, traduit le transfert de la chaleur produite éventuellement dans l'élément et l'accroissement de l'énergie interne.

Le calcul de la température moyenne suivant la direction considérée conduit à l'expression :

$$
(T)_{\mathrm{moy}}=\left(T_{\mathrm{DT}}\right)_{\mathrm{moy}}\left(T_{1}, T_{2}\right)+\left(T_{\mathrm{CP}}\right)_{\mathrm{moy}}(C, P)
$$

avec

$$
\left(T_{\mathrm{DT}}\right)_{\mathrm{moy}}=T_{1}-\frac{G_{x}}{G_{1}}\left(T_{1}-T_{2}\right)=T_{2}+\frac{G_{x}}{G_{2}}\left(T_{1}-T_{2}\right)
$$

et

$$
\left(T_{\mathrm{CP}}\right)_{\mathrm{moy}}=\frac{P-C \frac{\mathrm{d}(T)_{\mathrm{moy}}}{\mathrm{d} t}}{G_{3}^{\prime}}
$$

où

C

$P$

$G_{1}, G_{2}, G_{3}$ est la capacité calorifique de l'élément : $C=\rho C_{\mathrm{v}} \cdot \mathrm{d} x \mathrm{~d} y \mathrm{~d} z$. est la puissance produite dans l'élément : $P=p \cdot \mathrm{d} x \mathrm{~d} y \mathrm{~d} z$.

sont des grandeurs homogènes à des conductances thermiques (définies par analogie avec les conductances électriques par l'expression $G=k \frac{\mathrm{d} S}{\mathrm{~d} l}$ où $\mathrm{d} l$ est compté sur la direction normale à $\mathrm{dS}$ ).
$G_{x}=\frac{G_{1} G_{2}}{G_{1}+G_{2}}$ est la conductance thermique de l'élément suivant la direction $x$.

$\frac{\mathrm{d}(T)_{\text {moy }}}{\mathrm{d} t}$ la valeur uniforme de la dérivée de la température par rapport au temps.

On peut associer aux expressions de $\left(T_{\mathrm{DT}_{x}}\right)_{\text {moy }}$ et $\left(T_{\mathrm{CP}_{x}}\right)_{\text {moy }}$ deux schémas équivalents (Figs. 3c et $\mathrm{d}$ ). Ceci permet de déduire aisément un schéma équivalent pour le cas général (Fig. 3e). Ce schéma, en "étoile», est transformé en schéma en "triangle» (Fig. 3f) afin de supprimer le nœud $\mathbf{N}$.

L'expression analytique de $T(x)$ permet par ailleurs d'obtenir sans grande difficulté une relation permettant de déterminer la valeur maximale $\left(T_{x}\right)_{\max }$ suivant la direction considérée en fonction de $\left(T_{x}\right)_{\text {moy }}, T_{1}, T_{2}$ et des propriétés physiques.

Avec la représentation transformée de la figure $3 f$, nous voyons se préciser la notion de nouds à la périphérie sur les faces : les noeuds de liaisons. Les températures à ces nouds sont les températures moyennes sur les faces correspondantes de l'élément. Ce sont les températures des surfaces d'échanges lorsque les deux éléments reliés sont de natures différentes, l'un étant convectif au rayonnement. De plus ces températures de surfaces sont généralement accessibles expérimentalement, ce qui conforte leur intérêt comme résultats du modèle.

La température moyenne au noeud de l'élément permet de déterminer les valeurs moyennes des propriétés physiques, leurs variations étant pratiquement linéaires avec la température. On procède de manière identique pour la détermination des pertes.

2.1.2 Représentation unidirectionnelle multiple. Lorsque le transfert se fait dans plusieurs directions (cas général des éléments conductifs), une représentation simple se déduit aisément de la précédente par juxtaposition de plusieurs schémas unidirectionnels (Fig. 4). Ceci suppose validées deux hypothèses :

- indépendance des transferts directionnels,

- températures moyennes identiques dans toutes les directions.

La valeur maximale de la température dans l'élément peut être encore évaluée par calculs des valeurs maximales dans chacune des directions et sélection de la valeur supérieure aux autres.

\subsection{ModÉLISATION DES ÉLÉMENTS CONVECTIFS.}

Elément convectif volumique. - L'équation de bilan de puissance se déduit directement de l'équation aux différences finies (Fig. 5a).

$$
\begin{aligned}
\rho S_{\mathrm{C}} C_{\mathrm{P}} V\left[\left(T_{\mathrm{f}}^{\prime}\right)_{\mathrm{moy}}-\left(T_{\mathrm{f}}\right)_{\mathrm{moy}}\right] & = \\
= & \sum_{i=1}^{N} h_{i} \cdot S_{i}\left[T_{i}-\left(T_{\mathrm{f}}\right)_{\mathrm{moy}}\right]
\end{aligned}
$$



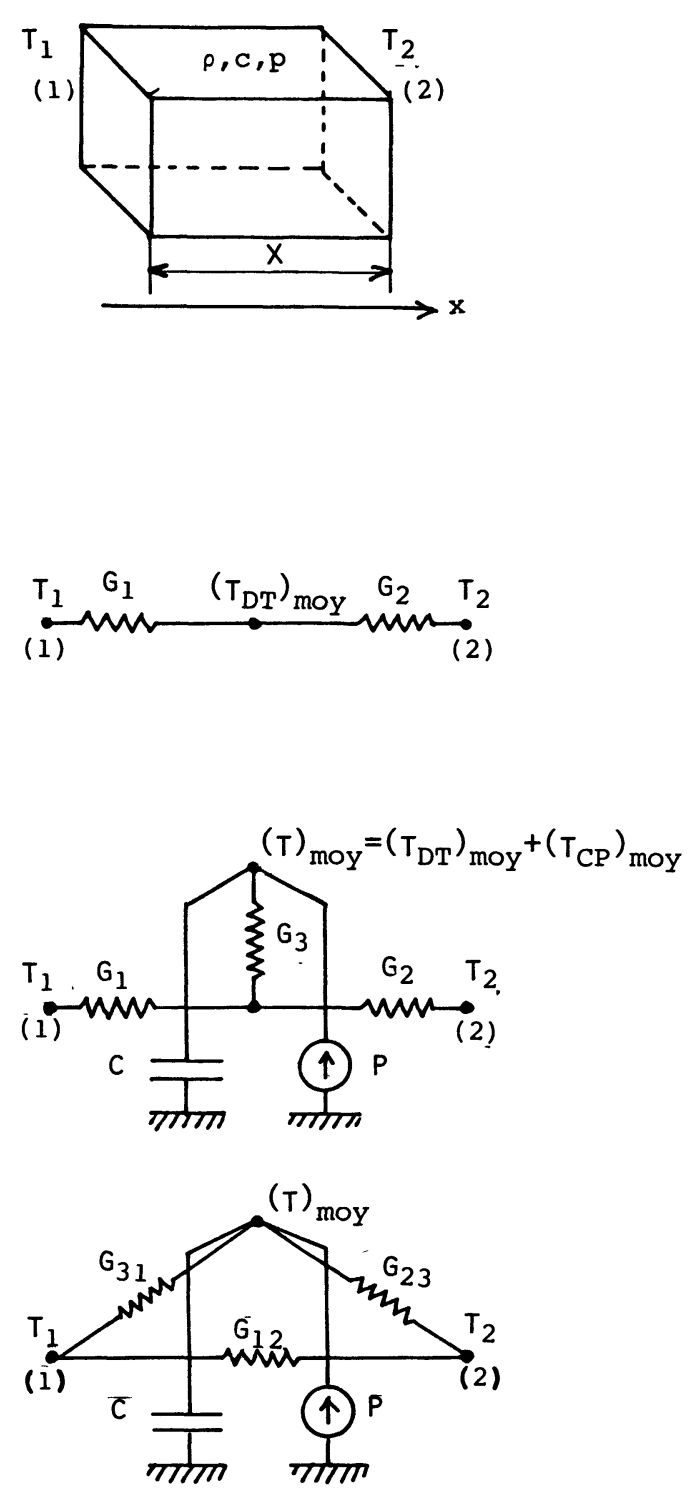
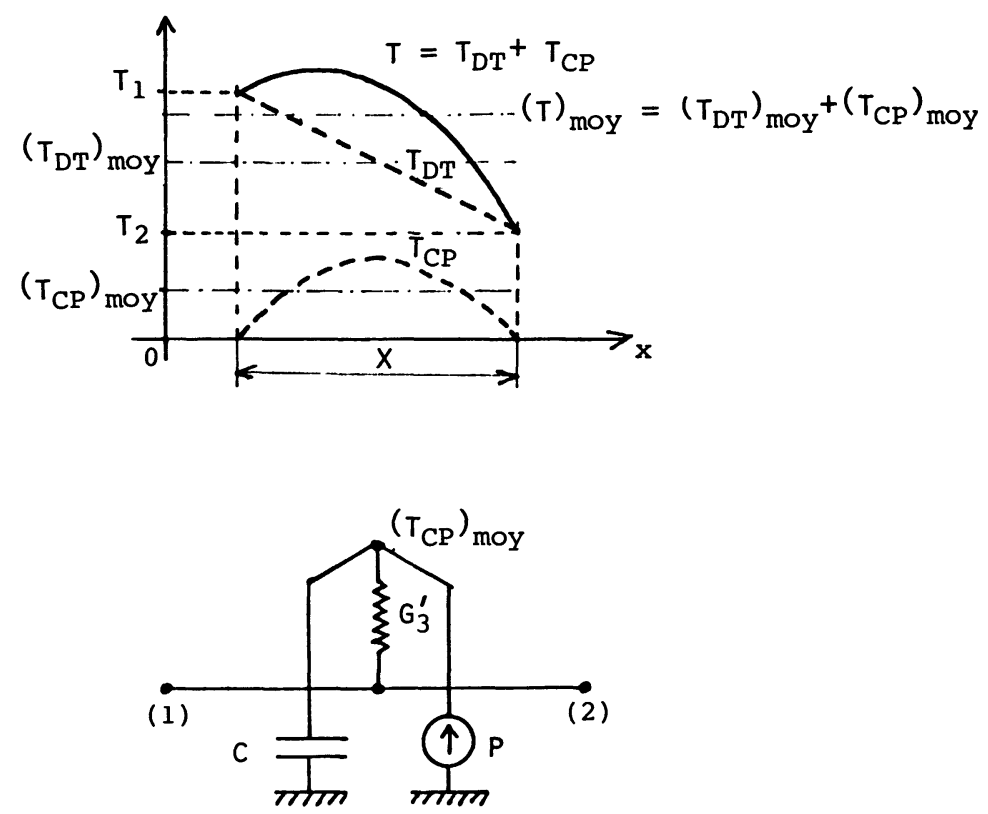

$$
G_{3}=\frac{G_{3}^{\prime}}{1-G_{3}^{\prime}\left(G_{1}+G_{2}\right)}
$$

$$
\begin{aligned}
& G_{31}=\frac{G_{3} \cdot G_{1}}{G_{1}+G_{2}+G_{3}} \\
& G_{12}=\frac{G_{1} \cdot G_{2}}{G_{1}+G_{2}+G_{3}} \\
& G_{23}=\frac{G_{2} \cdot G_{3}}{G_{1}+G_{2}+G_{3}}
\end{aligned}
$$

Fig. 3. - Mise en place du modèle élémentaire unidirectionnel.

[Unidirectional elementary model.]

où

$\left(T_{\mathrm{f}}\right)_{\mathrm{moy}}$, la température moyenne du fluide en mouvement dans l'élément considéré.

$\left(T_{\mathrm{f}}^{\prime}\right)_{\mathrm{moy}}, \quad$ la température moyenne du fluide dans l'élément aval.

$N, \quad$ le nombre de parois d'échanges à la périphérie de l'élément.

$T_{i}, S_{i}$ et $h_{i}$, la température, la surface et le coefficient d'échanges de la paroi $i$.

$\rho \quad$ est la masse volumique et $V$ la vitesse d'écoulement du fluide.

Elément convectif surfacique. - Dans un élément de ce type aucun déplacement général du fluide n'est distingué. Les transferts sont définis uniquement sur les parois qui délimitent l'enceinte contenant le fluide.
L'équation de transfert se déduit de la précédente par suppression du terme correspondant à l'écoulement :

$$
\sum_{i=1}^{N} h_{i} \cdot S_{i}\left[T_{i}-\left(T_{\mathrm{f}}\right)_{\mathrm{moy}}\right]=0 .
$$

On définit ainsi les conductances surfaciques :

$$
G_{i}=h_{i} \cdot S_{i} .
$$

La représentation schématique est reproduite sur la figure $5 b$.

2.3 ModÉLISATION DES ÉLÉMENTS RAYONNANTS. Pour un élément surfacique rayonnant, les transferts sont également définis aux surfaces frontières délimitant l'enceinte. 


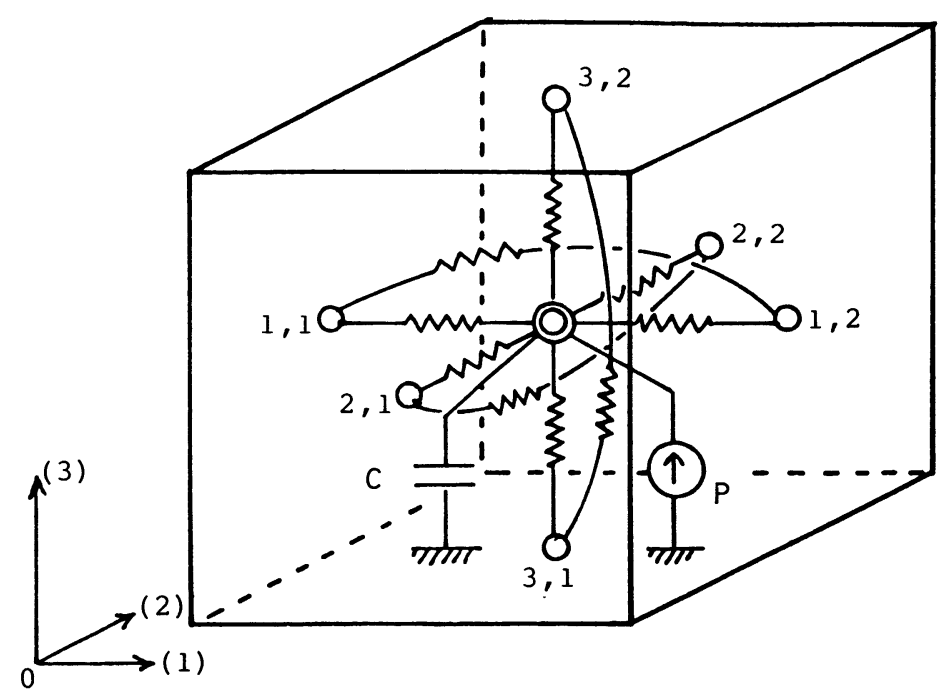

Fig. 4. - Représentation unidirectionnelle multiple. () Nœud de l'élément; 1,1 ○ nœud de liaison. [Unidirectional multiple representation. (O) Element node; $1,1 \circ$ link node.]

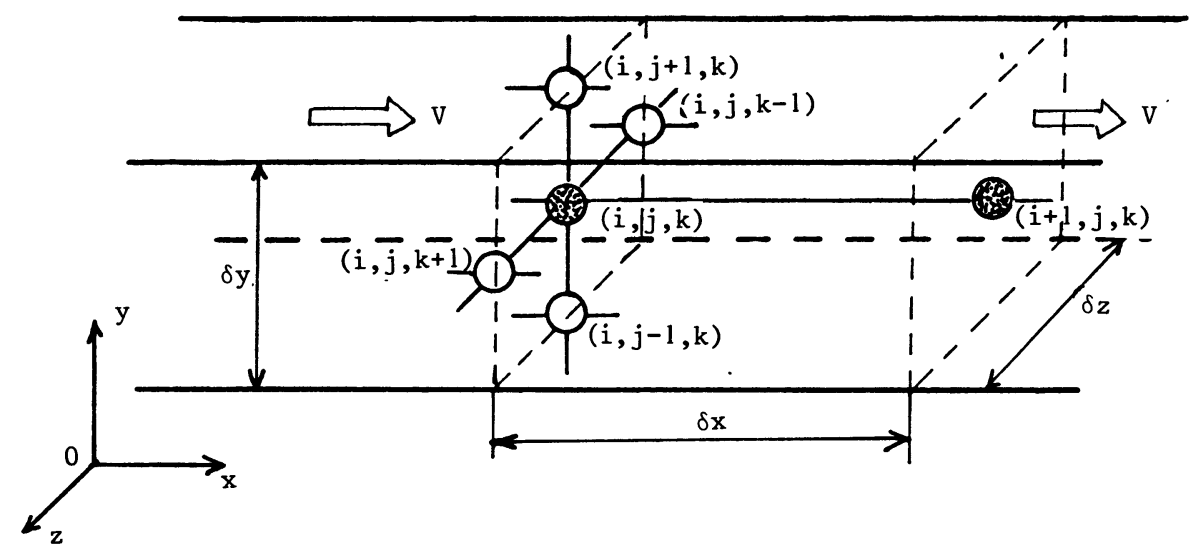

Fig. 5a. - Elément fluide en coordonnées cartésiennes. O Nœud solide des parois. Nœud fluide.

[Fluid element in cartesian coordinates. $\bigcirc$ Wall solid node. Fluid node.]

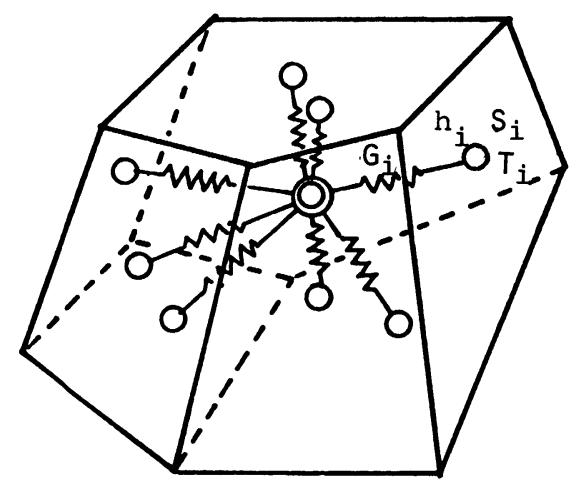

Fig. 5b. - Représentation schématique équivalente d'un élément surfacique correctif.

[Equivalent schematic representation of a convection surface element.]
Eléments rayonnants multiples. - Pour une face $(i)$ considérée mate et grise d'une telle enceinte délimitée par $N$ surfaces, le bilan de puissance s'écrit :

$$
P_{i}=S_{i}\left(\sum_{j=1}^{N} F_{i-j} \cdot p_{\mathrm{e}, j}-p_{\mathrm{e}, i}\right)
$$

avec la notation :

$$
F_{i-j}=\frac{1}{S_{i}} \int_{S_{i}} \int_{S_{j}} \frac{\cos \gamma_{i} \cos \gamma_{j} \cdot \mathrm{d} S_{i} \mathrm{~d} S_{j}}{\pi l^{2}}
$$

$F_{i-j} \quad$ est le facteur d'angles [21],

$\gamma_{i}=$ angle avec la normale à la surface $\mathrm{d} S_{i}$,

$l=$ distance des centres de $\mathrm{d} S_{i}$ et $\mathrm{d} S_{j}$,

$p_{\mathrm{e}, i}=$ est la densité de flux émis,

$p_{\mathrm{e}, i}=\varepsilon_{i} \sigma T_{i}^{4}+\mu_{i} P_{\mathrm{r}, i}$ 
$\mu_{i}=1-\varepsilon_{i}$ est le facteur de réflexion,

$P_{\mathrm{r}, i}=$ est la densité de flux reçu,

$\sigma \quad$ est la constante de Stephan.

La densité de flux d'énergie émis $p_{\mathrm{e}, i}$ peut s'écrire :

$$
p_{\mathrm{e}, i}=\sigma T_{i}^{4}+\frac{\mu_{i}}{\varepsilon_{i} S_{i}} P_{i}
$$

En appliquant la relation entre le facteur d'angle dans une enceinte, on obtient une nouvelle expression pour $P_{i}$ :

$$
P_{i}=\sum_{j=1}^{N}\left(p_{\mathrm{e}, j}-p_{\mathrm{e}, i}\right) S_{i} \cdot F_{i-j}
$$

On en déduit des égalités dont une représentation est donnée par le schéma équivalent électrique de la figure 6 , où les potentiels aux nœuds sont les termes $p_{\mathrm{e}, j}$ et $\sigma T_{j}^{4}$ :

$$
\left(\sigma T_{i}^{4}-p_{\mathrm{e}, i}\right) \frac{\varepsilon_{i} S_{i}}{\mu_{i}}=\sum_{j=1}^{N}\left(p_{\mathrm{e}, i}-p_{\mathrm{e}, j}\right) S_{j} \cdot F_{j-i} .
$$

La nature différente de l'équivalence conduit à considérer séparément ce type d'élément pour lequel une résolution spécifique est retenue.

Elément rayonnant simple (surfaces grises et mates). - La représentation, identique à celle de la convection, convient parfaitement :

$$
P_{1}=h_{\mathrm{RAY}_{12}} \cdot S_{1}\left(T_{2}-T_{1}\right)
$$

avec

$h_{\mathrm{RAY}_{12}}=\frac{\sigma \cdot F_{1-2}\left[T_{1}^{3}+T_{1}^{2} T_{2}+T_{1} T_{2}^{2}+T_{2}^{3}\right]}{1+\left[\frac{1-\varepsilon_{1}}{\varepsilon_{1}}+\frac{S_{1}}{S_{2}} \frac{1-\varepsilon_{2}}{\varepsilon_{2}}\right] F_{1-2}}$

ce qui permet de définir une conductance rayonnante.

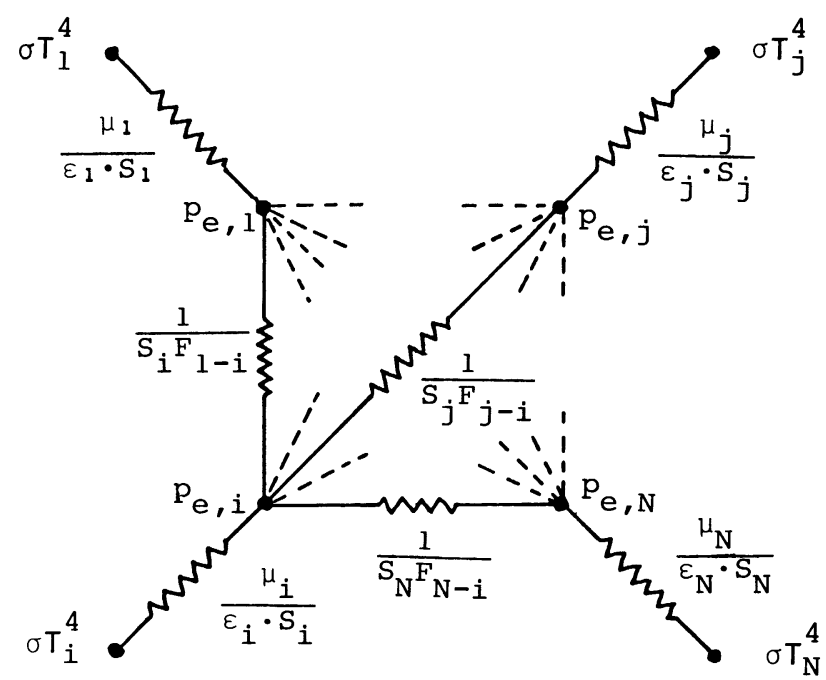

Fig. 6. - Représentation schématique équivalente du rayonnement multiple.

[Equivalent schematic representation of multiple radiation.]
2.4 MODÉLISATION DE L'ÉLÉMENT DE RÉFÉRENCE. Cet élément est à la fois rayonnant et convectif. Les surfaces extérieures d'une machine étant généralement convexes, on peut considérer que le rayonnement est simple, avec l'enceinte générale supposée noire. La représentation de l'élément se déduit donc des représentations précédentes (Fig. 7). L'équation de bilan s'écrit, si $N$ surfaces échangent avec l'extérieur :

$$
\sum_{i=1}^{N} S_{i}\left(h_{i, \mathrm{CONV}}+h_{i, \mathrm{RAY}}\right)\left(T_{\mathrm{A}}-T_{i}\right)=0
$$

où

$h_{i, \text { CoNv }}$ est le coefficient d'échanges par convection libre ou forcée;

$$
h_{i, \mathrm{RAY}}=\varepsilon_{i} \sigma\left(T_{\mathrm{A}}^{3}+T_{\mathrm{A}}^{2} T_{i}+T_{\mathrm{A}} T_{i}^{2}+T_{i}^{3}\right)
$$

est le coefficient d'échanges par rayonnement, $\varepsilon_{i}, S_{i}, T_{i}$ sont respectivement l'émissivité, l'aire et la température de la surface $S_{i}$,

$T_{\text {A }} \quad$ est la température supposée constante du milieu ambiant.

\section{Détermination des coefficients intervenant dans les équations de bilan.}

Ces coefficients sont les grandeurs définies lors de l'établissement des modèles élémentaires : les capacités calorifiques et les conductances thermiques.

Lorsque l'influence de la température est prise en compte, ces termes peuvent être présentés tous comme le produit d'une fonction des dimensions géométriques de l'élément représenté ou de la surface d'échange concernée et d'une fonction des propriétés physiques du matériau constitutif ou de la surface, dépendantes de la température. Généralement cette dépendance peut être décrite par une loi de variation linéaire compte tenu du domaine dans lequel se maintiennent les températures des machines électriques tournantes.

Dans le cas d'une conductance convective, la fonction des propriétés physiques correspond au coefficient

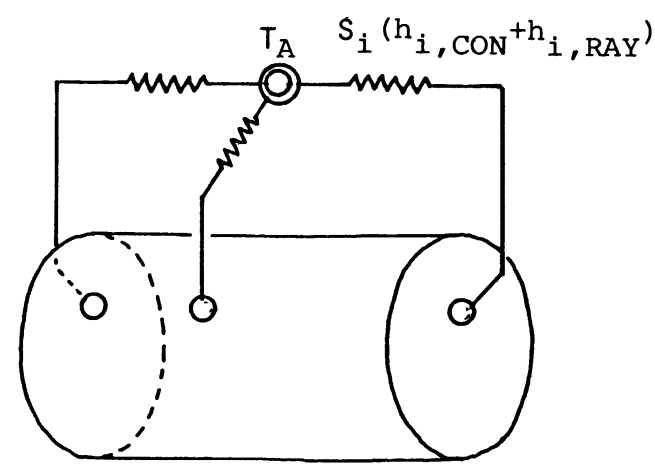

Fig. 7. - Représentation schématique équivalente de l'élément de référence.

[Equivalent schematic representation of reference element.] 
d'échange $h$ qui peut être déterminé, suivant la nature $\mathrm{du}$ transfert, par deux types de relations [22] :

- pour la convection forcée :

$$
h=\frac{(k)_{\mathrm{m}}}{L} \cdot a \cdot F\left(\frac{L}{d}\right)\left(N_{\mathrm{PR}}\right)_{\mathrm{m}}^{b^{\prime}}\left(N_{\mathrm{RE}}\right)_{\mathrm{m}}^{b},
$$

- pour la convection libre :

$$
h=\frac{(k)_{\mathrm{m}}}{L} \cdot a \cdot\left(\frac{L}{d}\right)^{c}\left(N_{\mathrm{PR}}\right)_{\mathrm{m}}^{b}\left(N_{\mathrm{GR}}\right)_{\mathrm{m}}^{b},
$$

où $N_{\mathrm{PR}}, N_{\mathrm{RE}}, N_{\mathrm{NU}}, N_{\mathrm{GR}}$ sont respectivement les nombres de Prandtl, Reynolds, Nüsselt et Grashof, $L$ une dimension linéaire caractéristique de la surface d'échange, $k$ la conductivité thermique de la couche limite du fluide, $a, b, b^{\prime}$ et $c$ des coefficients dépendant $\mathrm{du}$ mode d'écoulement, d'une dimension linéaire caractéristique du dispositif d'échange et $F(L / d)$ une fonction sans dimension permettant de prendre en compte les effets d'extrémité.

L'indice $\mathrm{m}$ indique que l'on prend des valeurs moyennes dans l'intervalle de température [20-24].

Lorsque l'air est le fluide réfrigérant :

- $(k)_{\mathrm{m}}$ approchée par une loi de la forme $k_{0}[1+$ $\left.\alpha_{\mathrm{k}}\left(T-T_{0}\right)\right]$,

- $\left(N_{\mathrm{PR}}\right)_{\mathrm{m}}$ est pratiquement constant, forme :

- $\left(N_{\mathrm{RE}}\right)_{\mathrm{m}}$ peut être approché par une loi de la

$$
\frac{a_{1} \cdot v L}{\left(T_{\mathrm{m}}+T_{\mathrm{C}_{1}}\right)^{b_{1}}}
$$

où $a_{1}, b_{1}$ et $T_{\mathrm{C}_{1}}$ sont des constantes; forme :

- $\left(N_{\mathrm{GR}}\right)_{\mathrm{m}}$ peut être approché par une loi de la

$$
\frac{a_{2} L^{3}\left|T_{\mathrm{S}}-T_{\mathrm{F}}\right|}{\left(T_{\mathrm{m}}+T_{\mathrm{C}_{2}}\right)^{b_{2}}}
$$

Les deux expressions de $h$ s'écrivent alors :

- pour la convection forcée :

$$
h=H k_{0}\left[1+\alpha_{\mathrm{k}}\left(T_{\mathrm{m}}-T_{0}\right)\right] \cdot\left[\frac{a_{1}}{\left(T_{\mathrm{m}}+T_{\mathrm{C}_{1}}\right)^{b_{1}}}\right]^{b}
$$

avec

$$
H=a L^{b-1} v^{b} F\left(\frac{L}{d}\right)\left(N_{\mathrm{PR}}\right)_{\mathrm{m}}^{b^{\prime}} ;
$$

- pour la convection libre :

$h=H k_{0}\left[1+\alpha_{\mathrm{k}}\left(T_{\mathrm{m}}-T_{0}\right)\right] \cdot\left[\frac{a_{2}}{\left(T_{\mathrm{m}}+T_{\mathrm{C}_{2}}\right)^{b_{2}}}\right]^{b}\left|T_{\mathrm{S}}-T_{\mathrm{F}}\right|^{b}$ avec

$$
H=a L^{3 b-1}\left(\frac{L}{d}\right)^{c}\left(N_{\mathrm{PR}}\right)_{\mathrm{m}}^{b}
$$

Dans ces expressions seuls $H$ et $b$ dépendent des conditions et des configurations d'échange. Les autres

termes dépendent des propriétés du fluide ou des températures.

Dans le cas d'une conductance rayonnante la fonction des propriétés physiques correspond au coefficient d'échange défini par les expressions (3) et (2) avec :

$$
\varepsilon_{i} \# \varepsilon_{i 0}\left[1+\alpha_{\varepsilon i}\left(T_{i}-T_{i 0}\right)\right] \quad i=1,2 .
$$

Les définitions des fonctions des dimensions géométriques et des fonctions des propriétés physiques, pour tous les coefficients des équations de bilan, sont rassemblées dans le tableau I.

\section{Systèmes d'équations des modèles thermiques.}

Ces équations sont les équations de bilan des puissances aux différents nouds définis par le découpage. Les variables d'état sont les écarts de température par rapport à celle de l'ambiance, supposée fixe, prise comme référence.

Deux types de nouds ont été définis :

- les noeuds de liaisons dont les équations de bilan correspondent aux conditions aux limites des éléments adjacents ;

- les noeuds d'éléments, uniquement d'éléments " matériels », conductifs et convectifs, dont les équations de bilan correspondent aux équations de transfert dans les milieux considérés.

Les termes correspondant au transfert par rayonnement interviennent dans les équations de bilan des nouds de liaison associés aux surfaces échangeant suivant ce mode.

Considérons un modèle comportant :

- $\mathrm{N}_{1}$ nouds de liaisons

- $\mathrm{N}_{2}$ noeuds d'éléments convectifs

- $\mathrm{N}_{3}$ noeuds d'éléments conductifs

- $\mathrm{N}_{4}$ éléments rayonnants.

- L'équation d'un noeud de liaisons $l$ peut prendre la forme symbolique suivante, certains termes s'excluant :

$$
\begin{aligned}
\sum_{l^{\prime}=1}^{M_{1 \mathrm{~L}}} G_{l l^{\prime}}\left(\theta_{l}-\theta_{l^{\prime}}\right)+ & \sum_{i=1}^{M_{1 \mathrm{c}}} G_{l i}\left(\theta_{l}-\theta_{i}\right)+ \\
& +\sum_{j=1}^{M_{1 \mathrm{D}}} G_{l j}\left(\theta_{l}-\theta_{j}\right)+G_{l \mathrm{~A}} \theta_{l}=P_{l}
\end{aligned}
$$

où

$M_{1 \mathrm{~L}}, M_{1 \mathrm{C}}$ et $M_{1 \mathrm{D}}$ sont respectivement les nombres de nouds de liaisons, d'éléments conductifs et d'éléments convectifs liés à $l$.

$G_{l l^{\prime}}$ et $G_{l i}$

$G_{l j}$

$G_{l \mathbf{A}}$

$P_{l}$ des conductances conductives.

des conductances convectives surfaciques ou fluides.

une conductance radiative et convective de l'élément de référence. la puissance reçue par rayonnement. 
Tableau I. - Détermination des coefficients des équations des modèles. La température $T_{0}$ est choisie égale à $T_{\mathrm{A}}$ température de l'ambiance pour toutes les grandeurs.

\begin{tabular}{|c|c|c|c|c|}
\hline \multicolumn{2}{|c|}{ Nature des coefficients } & $\begin{array}{l}\text { Fonctions des dimensions } \\
\text { géométriques }\end{array}$ & $\begin{array}{l}\text { Fonctions des propriétés } \\
\text { physiques }\end{array}$ & Données \\
\hline \multirow{5}{*}{ Conductances } & conductives & $\begin{array}{l}\text { dépend de la forme de l'élé- } \\
\text { ment }\end{array}$ & $k=k_{0}\left(1+\alpha_{k}\left(T-T_{0}\right)\right)$ & $k_{0}, \alpha_{\mathrm{k}}$ \\
\hline & fluides & $\begin{array}{l}\quad S . v \\
S \text {, section du canal } \\
v, \text { vitesse écoulement }\end{array}$ & $\begin{array}{c}\rho . C \\
\text { avec } C=C_{0}\left(1+\alpha_{C}\left(T-T_{0}\right)\right)\end{array}$ & $\begin{array}{c}\rho \\
C_{0}, \alpha_{\mathrm{C}}\end{array}$ \\
\hline & $\begin{array}{l}\text { convectives } \\
\text { surfaciques }\end{array}$ & $\begin{array}{c}S \\
\text { aire de la surface d'échange }\end{array}$ & $\begin{array}{l}h \\
\text { en convection forcée par } \\
\text { (II.18a et b), en convection } \\
\text { libre par (II.19a et b) }\end{array}$ & $\begin{array}{c}\text { nature de la } \\
\text { convection, } \\
H, b\end{array}$ \\
\hline & \multirow[t]{2}{*}{ rayonnantes } & \multirow{2}{*}{$\begin{array}{l}S_{1} \\
\text { aire d'une des surfaces d'é- } \\
\text { change }\end{array}$} & $\begin{array}{l}\text { cas de deux surfaces grises } \\
\text { et mates: } \\
\qquad h_{\mathrm{RAY}_{12}} \\
\text { par (II.13) avec (II.20) }\end{array}$ & $\begin{array}{l}F_{1-2} \\
\varepsilon_{1}, \alpha_{\varepsilon 1} \\
\varepsilon_{2}, \alpha_{\varepsilon 2}\end{array}$ \\
\hline & & & $\begin{array}{l}\text { cas où l'autre surface est } \\
\text { noire : } \\
\quad h_{1, \mathrm{RAY}} \\
\operatorname{par}(\text { II. 15) avec (II.20) }\end{array}$ & $\begin{array}{c}\rho \\
\varepsilon_{1}, \alpha_{\varepsilon 1}\end{array}$ \\
\hline \multicolumn{2}{|c|}{ Capacités } & volume $v$ & $\begin{array}{c}\rho . C \\
\operatorname{avec} C=C_{0}\left(1+\alpha_{\mathrm{C}}\left(T-T_{0}\right)\right)\end{array}$ & $\begin{array}{c}\rho \\
C_{0}, \alpha_{\mathrm{C}}\end{array}$ \\
\hline
\end{tabular}

Le terme correspondant à l'élément de référence interdit bien sûr à la fois le terme correspondant au rayonnement et celui correspondant à la convection.

La puissance reçue par rayonnement est calculée différemment selon que le rayonnement est simple ou multiple :

- Dans le cas du rayonnement simple $P_{l}$ est calculée à partir d'une expression de la forme :

$$
P_{l}=G_{\mathrm{RAY}_{l l^{\prime}}}\left(\theta_{l^{\prime}}-\theta_{l}\right)
$$

qui définit une conductance $G_{\mathrm{RAY}_{u^{\prime}}}$ qui peut être regroupée avec les 'conductances $G_{l l^{\prime}}$ entre nouds de liaisons.

- Dans le cas du rayonnement multiple $P_{l}$ est calculée à partir d'une expression de la forme :

$$
P_{l}=\sum_{j=1}^{M_{4 L}}\left(p_{\mathrm{e}, j}-p_{\mathrm{e}, l}\right) S_{l} \cdot F_{l-j}
$$

où $M_{4 \mathrm{~L}}$ est le nombre de surfaces rayonnantes de l'élément concerné.

Les densités surfaciques de flux d'énergie $p_{e, j}$ et $p_{\mathrm{e}, l}$ sont les solutions du système de $M_{4 \mathrm{~L}}$ équations établi à partir des équations du type de (1) en appli- quant les règles de réciprocité :

$$
\sum_{k=1}^{M_{42}}\left(\frac{\delta_{l, k}}{\mu_{l}}-F_{l-k}\right) \cdot p_{\mathrm{e}, k}=\frac{\varepsilon_{l}}{\mu_{l}} \sigma T_{l}^{4}
$$

pour $l=1$ à $M_{4 \mathrm{~L}}$, avec

$$
\begin{aligned}
& \left\{\begin{array}{lll}
\delta_{l k}=1 & \text { si } & l=k \\
\delta_{l k}=0 & \text { si } & l \neq k
\end{array}\right. \\
& T_{l}=\theta_{l}+T_{\mathrm{A}}
\end{aligned}
$$

où $T_{\mathrm{A}}$ est la température de la référence (milieu ambiant).

- L'équation d'un noud convectif $j$ prend la forme suivante :

$$
\sum_{l=1}^{M_{2 L}} G_{j l}\left(\theta_{j}-\theta_{l}\right)=0
$$

où

$M_{2 \mathrm{~L}}$ est le nombre de nœuds de liaisons périphériques de l'élément $j$.

$G_{j l}$ sont des conductances convectives surfaciques ou fluides. 
- L'équation d'un noud conductif $i$ prend la forme :

$$
\sum_{l=1}^{M_{3 L}} G_{i l}\left(\theta_{i}-\theta_{l}\right)+C_{i} \frac{\mathrm{d} \theta_{i}}{\mathrm{~d} t}=P_{i}
$$

où

$M_{3 \mathrm{~L}}$ est le nombre de nœuds de liaisons périphériques de l'élément $i$.
$G_{i l}$ des conductances conductives de l'élément.

$C_{i} \quad$ la capacité calorifique.

$P_{i} \quad$ la puissance produite éventuellement dans l'élément.

Les équations du modèle sont ensuite regroupées dans deux systèmes dont les formes symboliques sont les suivantes :
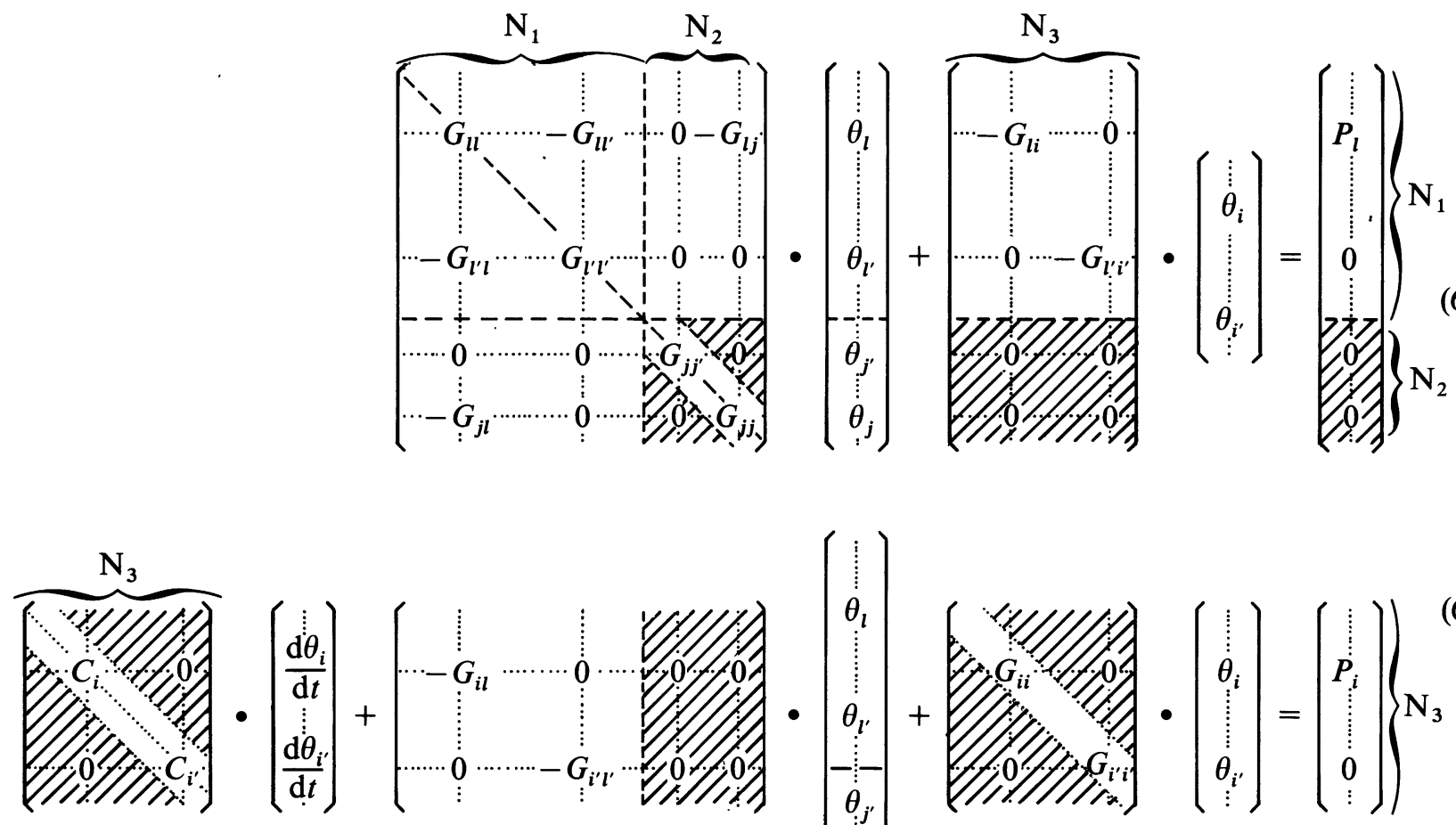

$\left(\begin{array}{c}\vdots \\ \vdots \\ \theta_{l} \\ \vdots \\ \vdots \\ \theta_{l^{\prime}} \\ \vdots \\ \hdashline \vdots \\ \theta_{j^{\prime}} \\ \vdots \\ \theta_{j}^{j} \\ \vdots\end{array}\right)$
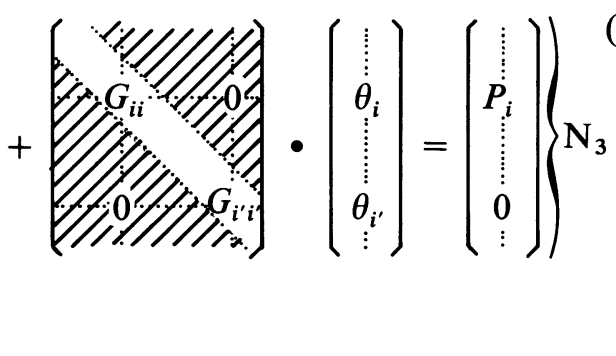

Note : les parties hachurées des matrices ne contiennent que des éléments nuls.

- le système algébrique (6a),

- le système différentiel (6b).

Les différentes matrices de conductance de ces systèmes comportent de nombreux éléments nuls. Dans ces matrices les éléments obéissent à la règle suivante :

$$
G_{i j}=G_{j i} \quad \forall i \text { et } j .
$$

Les conductances propres de nouds, éléments des diagonales des matrices carrées, sont calculées à partir des relations suivantes :

— pour les nœuds de liaisons :

$$
G_{l l}=\sum_{l^{\prime}=1}^{M_{1 \mathrm{~L}}} G_{l l^{\prime}}+\sum_{i=1}^{M_{1} \mathrm{C}} G_{l i}+\sum_{j=1}^{M_{1 \mathrm{D}}} G_{l j}+G_{l \mathrm{~A}}
$$

où $G_{I \mathrm{~A}}$, conductance de l'élément de référence exclut les conductances convectives $G_{l j}$ et toute conductance rayonnante parmi les $G_{l l^{\prime}}$
- pour les nœuds d'éléments convectifs :

$$
G_{j j}=\sum_{l=1}^{M_{2 L}} G_{j l}
$$

- pour les nouds d'éléments conductifs :

$$
G_{i i}=\sum_{l=1}^{M_{3 L}} G_{i l} .
$$

Les deux systèmes (6a) et (6b) sont résolus séparément. Si le modèle comporte des éléments rayonnants multiples, le mode de transfert par rayonnement n'étant pas prépondérant dans une machine électrique tournante, on peut procéder comme suit :

- Les écarts de températures $\theta_{l}, \theta_{i}, \theta_{i^{\prime}}, \ldots$, sont calculés par résolution des systèmes $(6 a)$ et $(6 b)$ en supposant tous les flux rayonnants $P_{l}$ nuls.

- A partir de ces premières valeurs des écarts de températures on évalue les flux rayonnants $P_{l}$ à 


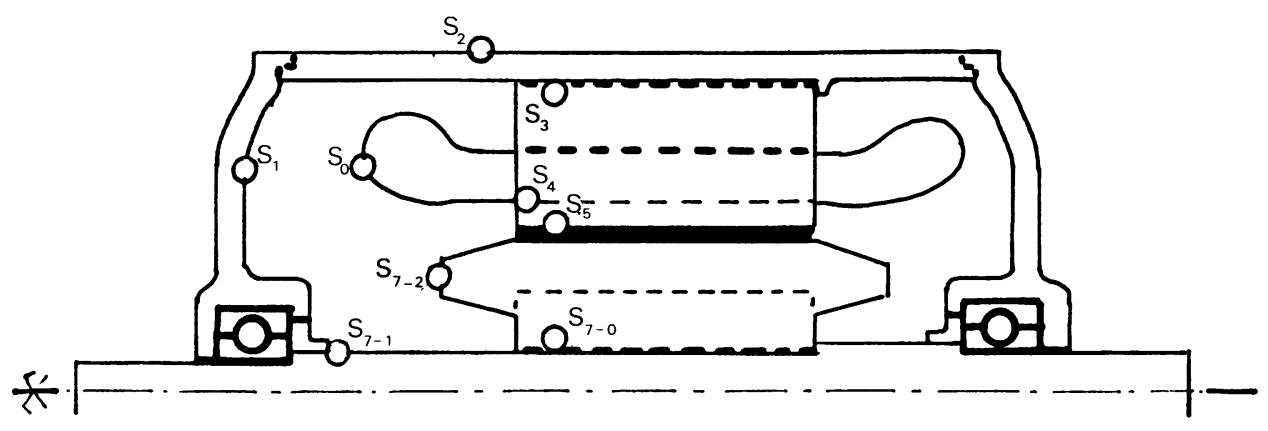

Fig. 8. - Emplacement des sondes thermiques.

[Location of temperature probes.]

partir des relations (4) après résolution du système (5) pour chacun des éléments rayonnants multiples.

- En portant ces valeurs des $P_{l}$ dans le système (6a) on le résout à nouveau ainsi que le système (6b) obtenant de nouvelles valeurs des écarts des températures.

- Cette procédure est réitérée jusqu'à ce que les différences entre les valeurs successives des écarts de températures soient inférieures ou égales à la précision désirée.

\section{Application de cette modélisation à une machine asynchrone de faible puissance. Résultats et conclu- sions.}

La machine de test est un moteur asynchrone de $700 \mathrm{~W}$ autoventilé et fermé. Elle est munie de sondes thermiques permettant la mesure de la température en divers points du stator et du rotor figure 8.

La première difficulté rencontrée est le manque de précision sur certaines dimensions constructives (épaisseur des isolants...) ou sur certains coefficients d'échange. Un essai d'identification au refroidissement a permis de fixer les valeurs mal connues à l'intérieur d'une fourchette de détermination. Elles sont ensuite maintenues constantes.

Les résultats obtenus apparaissent figure 9 lors d'un essai en frein et figure 10 pour un transitoire en court-circuit. (Les résultats expérimentaux sont connus à $\pm 1^{\circ}$.)

En conclusion, nous constatons donc une très bonne concordance entre les résultats expérimentaux et ceux donnés par le modèle thermique.

Il reste bien sûr le problème important des valeurs à donner à certains coefficients et échanges. Dans cet article, nous avons contourné la difficulté grâce à un essai d'identification. Lorsque ce n'est pas possible (par exemple lors de la prédétermination d'un prototype), le concepteur doit utiliser les valeurs moyennes données dans la littérature technique (ces valeurs varient d'un auteur à l'autre) ou faire appel à son expérience. Les résultats obtenus peuvent alors varier dans une fourchette de $10 \%$.

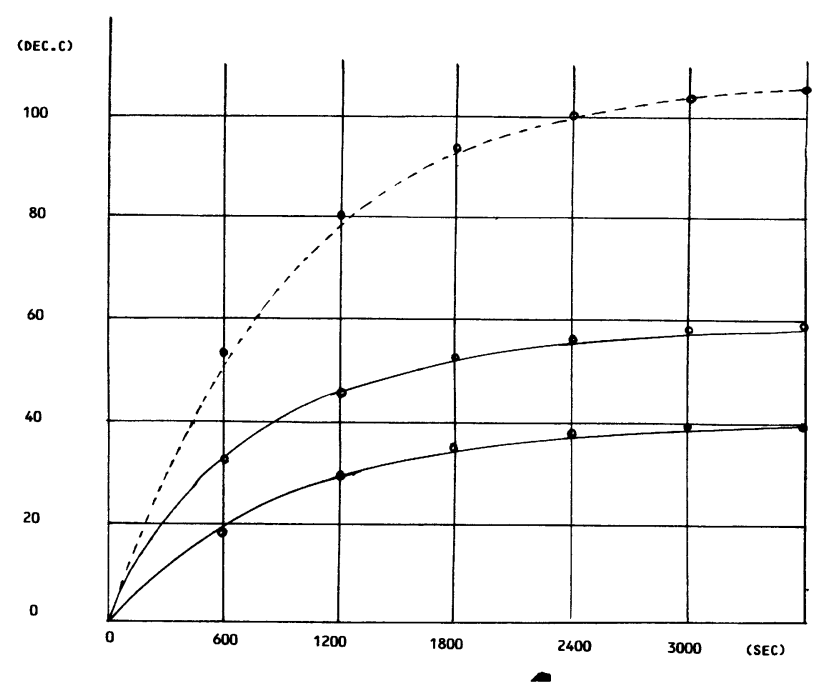

Fig. 9. - Transitoire thermique en fonctionnement en frein. ----- Résultat de la simulation; ..... Relevés expérimentaux.

[Thermal transient for braking operation. =---- Simulation results ;.... Experimental results.]

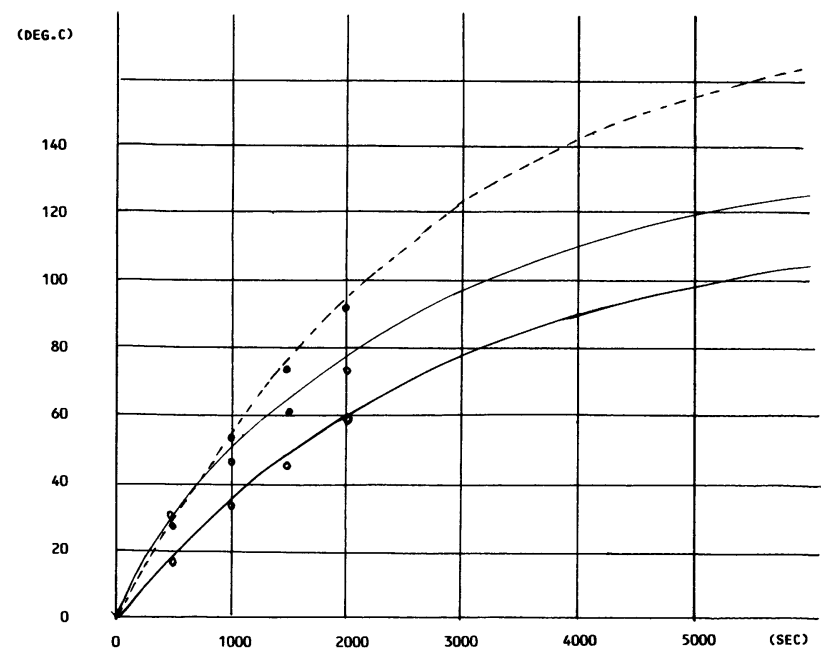

Fig. 10. - Transitoire thermique en court-circuit.

[Thermal transient for short-circuit.] 
Cette étude qui donne donc de bons résultats dans le cas d'un type de machine a l'avantage d'être très souple à l'emploi et facilement généralisable à d'autres machines. Elle constitue à notre avis un élément intéressant d'un logiciel de Conception Assistée par Ordinateur des machines électriques.

\section{Bibliographie}

[1] Chauprade, R., Milsant, F., Commande électronique des moteurs à courant alternatif (Editions Eyrolles) 1980.

[2] Wiart, A., Association d'onduleurs et de machines asynchrones, Colloque SEE-Marseille, 25-26 avril 1974.

[3] KIENER, A., Etude et réalisation d'un moteur synchrone à aimants Samarium-Cobalt, Thèse de DocteurIngénieur, INP Grenoble, juin 1981.

[4] Rioux, C., Faucher, J., Lucidarne, J., Sargos, F. M., Kauffmann, J. M., MaIlfert, A., Adaptation des machines à réluctance variable aux systèmes d'entraînements. Proceedings Colloque Européen : Au Carrefour de la Force-motrice, Paris, 10-11 décembre 1981.

[5] Bahbout, S., Roye, D., Perret, R., Heating of a static inverter fed induction machine, Congrès IEEE/IAS, Cincinnati 1980.

[6] GRellet, G., Etude générale de l'effet de saturation sur le fonctionnement des moteurs d'induction alimentés en onde non sinusoïdale, Thèse de Docteur ès Sciences Physiques, Ecole Centrale de Lyon, mai 1977.

[7] BuRAIS, N., Etude et modélisation des pertes dans les circuits magnétiques en régime non sinusoïdal à fréquences industrielles élevées, Thèse de DocteurIngénieur, Ecole Centrale de Lyon, mai 1981.

[8] GuREVIC, E. I., RyBIN, J. L., Les erreurs de la méthode thermométrique de mesures des pertes locales dans les machines électriques (en russe), Traduction $478 \mathrm{CF}, 1977$.

[9] BAнвоUт, S., Etude en régime permanent d'une machine asynchrone alimentée par un onduleur à transistors : performances, pertes, échauffements, Thèse de Docteur-Ingénieur, INP Grenoble, octobre 1981.

[10] OlIVIER, E., Etude des performances d'un moteur asynchrone à cage alimenté par un onduleur à transistors MOS de puissance commandé en modulation de largeur d'impulsions, Thèse de Docteur 3e Cycle, INP Grenoble, octobre 1982.
[11] Obenhaus, R. E., Sensor retrofitting of motors for protection against over-temperature, IEEE Trans. Industry Appl. IA-11, № 1, (1975) 24-33.

[12] Sheffert, K. W., Graham, D. L., Obenhaus, R. E., Application of inherent thermal protection to industrial motor systems, IEEE Trans. Industry Appl. IA-11, № 1, (1975) 14-23.

[13] Boothman, D. R., Elgar, E. C., Rehder, R. H., WoOdDALL, R. J., Thermal tracking : a rational approach to motor protection, IEEE PES (1974) 1335-1344.

[14] Martiny, W. T., McCoy, R. M., Margolis, H. B., Thermal relationships in an induction motor under normal and abnormal operation, AIEE Trans. Power Apparatus Systems (1961) 66-76.

[15] NaILEN, R. L., Safe locked rotor time : how safe is it? IEEE Trans. Industry Appl. IGA-7, № 6 (1971) 708-712.

[16] Kostenko, M., Piotrovski, L., Machines Electriques (Editions MIR, Moscou) 1969.

[17] Liwschitz, M., MARET, L., Calcul des machines électriques (Editions SPES, Lausanne) 1967.

[18] Normes, C 51-111, Règles d'Etablissement des Machines Electriques Tournantes, Novembre 1975.

[19] Marty, M., Contributions à l'étude de l'échauffement des machines électriques tournantes, Thèse Doctorat ès Sciences Physiques, Toulouse, avril 1965.

[20] Roye, D., Modélisation thermique des machines électriques tournantes. Application à la machine à induction. Thèse D.E., L.E. Grenoble 1983.

[21] General Electric Company, Heat Transfer and Fluid Flow Data Book.

[22] McAdams, W. H., Heat Transmission (McGraw Hill Book Company Inc.) 1954.

[23] KAYs, W. M., BJORKLUND, I. S., Heat transfer from a rotating cylinder with and without cross-flow; Transactions of ASME (1958) 70.

[24] Dropkin, D., CARMI, A., Natural convection from an horizontal surface rotating in air. Heat Transfer and Fluid Mechanism Institute (1956) 151. 